\title{
Crystal Structure of Moracin M
}

\section{Gilbert Deccaux KaPChe, ${ }^{* 1}$ Pierre Waffo-Teguo, ${ }^{* 2 \dagger}$ Stéphane MassiP, ${ }^{* 3}$ Jean GuILlon, ${ }^{* 3}$ Caroline VITRAC, ${ }^{* 2}$ Stéphanie KRISA, ${ }^{* 2}$ Bonaventure NGADJUI, ${ }^{* 4}$ and Jean-Michel MerILLON*2}

\author{
*1 Department of Chemistry, Higher Teachers' Training College, University of Yaounde, P.O. Box 47, \\ Yaounde, Cameroon \\ *2 GESVAB, EA 3675, ISVV, UFR des Sciences Pharmaceutiques, Université Victor Segalen Bordeaux 2, \\ 146 rue Léo Saignat, 33076 Bordeaux Cedex, France \\ *3 EA 2962 - Pharmacochimie, UFR des Sciences Pharmaceutiques, Université Victor Segalen \\ Bordeaux 2, 146 rue Léo Saignat, 33076 Bordeaux Cedex, France \\ *4 Department of Organic Chemistry, Faculty of Science, University of Yaounde I, P.O. Box 812, \\ Yaounde, Cameroon
}

\begin{abstract}
The structure of Moracin M, a stilbenoid extracted from the stem bark of Milicia excelsa (Moraceae), was established by a single-crystal X-ray analysis. It crystallizes in the monoclinic space group $P 2_{1} / n$. Solid-state data could be used to enlighten the biological mechanism of action.
\end{abstract}

(Received November 24, 2006; Accepted February 14, 2007; Published on web April 11, 2007)

\begin{abstract}
Milicia excelsa (Moraceae), commercially known as Iroko, is a valuable hardwood from humid tropics of Africa. ${ }^{1}$ Parts of this plant are employed in ethnomedicine to facilitate lactation after childbirth, for the treatment of ocular illnesses, and as a fungicide. $^{2}$ Iroko was further selected in the framework of a screening program to discover novel anticancer agents from Cameroonian medicinal plants.
\end{abstract}

During a phytochemistry investigation of the stem bark of Milicia excelsa (Moraceae) collected in Cameroon in 2006, we isolated a major stilbenoid, which was determined to be 2-( $3^{\prime}, 5^{\prime}-$ dihydroxyphenyl)-6-benzofuran (Fig. 1), previously identified as Moracin M, and isolated from various Morus species. ${ }^{3}$ We recently submitted this natural compound to an in vitro evaluation of its cytotoxic activity against $\mathrm{CaCo}-2$ cancer cells. It showed an $\mathrm{IC}_{50}$ of $70 \mu \mathrm{M}$ that was very close to the value of trans-resveratrol used as a reference drug $\left(\mathrm{IC}_{50}=62 \mu \mathrm{M}\right){ }^{4}$

Moracin $\mathrm{M}$ was isolated as follows: air-dried and finely powdered bark $(2 \mathrm{~kg}$ ) was macerated in methanol for $48 \mathrm{~h}$ at room temperature, followed by filtration and concentration to yield a crude methanol extract (150 g), which was washed with hexane and extracted with ethyl acetate. The ethyl acetate extract (50 g) was separated over a silica-gel chromatographic column using a gradient of $\mathrm{CH}_{3} \mathrm{Cl}-\mathrm{MeOH}$ (from 100/0 to 50/50) as an eluent. Fractions obtained $(15.5 \mathrm{~g})$ with $\mathrm{CH}_{3} \mathrm{Cl}-\mathrm{MeOH}$ (85/15) were re-chromatographied with $\mathrm{CH}_{3} \mathrm{Cl}-\mathrm{MeOH}(95 / 5)$ as an eluent to give the stilbenoid Moracin M (20 mg). Pale-

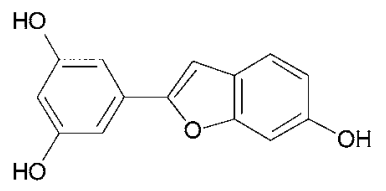

Fig. 1 Chemical structure of Moracin M.

$\doteqdot$ To whom correspondence should be addressed. E-mail: pierre.waffoteguo@u-bordeaux2.fr brown crystals suitable for X-ray analysis were obtained by slow evaporation from a chloroform-methanol solution.

The structure of Moracin M is illustrated in Fig. 2. Crystal and experimental data are given in Table 1.

As shown in Fig. 2, the benzofuranyl ring system is planar;

Table 1 Crystal and experimental data

Formula: $\mathrm{C}_{14} \mathrm{H}_{10} \mathrm{O}_{4}$

Formula weight: 242.22

Crystal system: monoclinic $\quad Z=4$

Space group: $P 2_{1} / n$

$a=13.319(4) \AA$

$b=5.415(5) \AA$

$c=15.927(4) \AA$

$\beta=109.17(2)^{\circ}$

$V=1085.0(11) \AA^{3}$

Crystal size $=0.25 \times 0.15 \times 0.03 \mathrm{~mm}^{3}$

$D_{\mathrm{x}}=1.483 \mathrm{Mg} / \mathrm{m}^{3}$

$F\left(\begin{array}{llll}0 & 0 & 0\end{array}\right)=504$

$\mu=0.915 \mathrm{~mm}^{-1}$

Temperature $=296(2) \mathrm{K}$

$\theta_{\max }=64.91^{\circ}$

Radiation: $\mathrm{Cu} K_{\alpha}(\lambda=1.54180 \AA)$

No. of reflections measured $=1844$

No. of reflections used $=1448$ with $I>2 \sigma(I)$

$R 1=0.0411$

$w R=0.0982$

Goodness-of-fit $=1.065$

$(\Delta / \sigma)_{\max }=0.049$

$(\Delta \rho)_{\max }=0.162 \mathrm{e} \AA^{-3}$

$(\Delta \rho)_{\min }=-0.169$ e $\AA^{-3}$

Measurement: Enraf-Nonius CAD4 ${ }^{5}$

Structure determination: SHELXS-97

Refinement: SHELXL-977

Crystallographic Data Centre as supplementary publication number CCDC-628349. Cambridge Crystallographic Data Centre, University Chemical Lab, 12 Union Road, Cambridge, CB2 1EZ, UK; E-mail: deposit@ccdc.cam.ac.uk. 


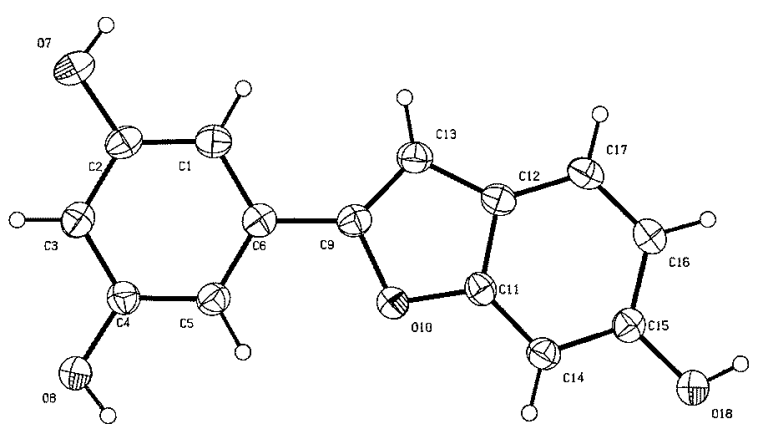

Fig. 2 Molecular structure of Moracin $M$ with our numbering scheme. Displacement ellipsoids are drawn at the $30 \%$ probability level.

Table 2 Final atomic coordinates and equivalent isotropic thermal parameters $\left(U_{\text {eq }}\left(\AA^{2}\right)\right)$

\begin{tabular}{ccccc}
\hline Atom & $x$ & $y$ & $z$ & $U_{\text {eq }}$ \\
\hline $\mathrm{C}(1)$ & $0.0733(1)$ & $-0.1615(4)$ & $0.8031(1)$ & $0.048(1)$ \\
$\mathrm{C}(2)$ & $0.0210(1)$ & $-0.1333(4)$ & $0.8640(1)$ & $0.049(1)$ \\
$\mathrm{C}(3)$ & $-0.0502(1)$ & $0.582(4)$ & $0.8569(1)$ & $0.050(1)$ \\
$\mathrm{C}(4)$ & $-0.0691(1)$ & $0.2207(4)$ & $0.7865(1)$ & $0.046(1)$ \\
$\mathrm{C}(5)$ & $-0.0160(1)$ & $0.2006(3)$ & $0.7258(1)$ & $0.045(1)$ \\
$\mathrm{C}(6)$ & $0.0553(1)$ & $0.0071(3)$ & $0.7329(1)$ & $0.043(1)$ \\
$\mathrm{O}(7)$ & $0.0358(1)$ & $-0.2863(3)$ & $0.9352(1)$ & $0.071(1)$ \\
$\mathrm{O}(8)$ & $-0.1437(1)$ & $0.4012(3)$ & $0.7797(1)$ & $0.060(1)$ \\
$\mathrm{C}(9)$ & $0.1085(1)$ & $-0.0239(3)$ & $0.6669(1)$ & $0.043(1)$ \\
$\mathrm{O}(10)$ & $0.0864(1)$ & $0.1573(2)$ & $0.6015(1)$ & $0.046(1)$ \\
$\mathrm{C}(11)$ & $0.1415(1)$ & $0.0925(3)$ & $0.5460(1)$ & $0.041(1)$ \\
$\mathrm{C}(12)$ & $0.1989(1)$ & $-0.1236(3)$ & $0.5754(1)$ & $0.044(1)$ \\
$\mathrm{C}(13)$ & $0.1751(1)$ & $-0.1942(3)$ & $0.6538(1)$ & $0.049(1)$ \\
$\mathrm{C}(14)$ & $0.1412(1)$ & $0.2216(3)$ & $0.4711(1)$ & $0.046(1)$ \\
$\mathrm{C}(15)$ & $0.2026(1)$ & $0.1245(3)$ & $0.4244(1)$ & $0.044(1)$ \\
$\mathrm{C}(16)$ & $0.2621(1)$ & $-0.908(4)$ & $0.4519(1)$ & $0.048(1)$ \\
$\mathrm{C}(17)$ & $0.2605(1)$ & $-0.2157(4)$ & $0.5267(1)$ & $0.050(1)$ \\
$\mathrm{O}(18)$ & $0.2045(1)$ & $0.2486(3)$ & $0.3498(1)$ & $0.055(1)$ \\
\hline
\end{tabular}

$U_{\text {(eq) }}=(1 / 3) \Sigma_{i} \Sigma_{j} U_{i j}\left(a_{i}^{*} a_{j}^{*}\right)\left(\boldsymbol{a}_{i} \cdot \boldsymbol{a}_{j}\right)$.

the maximum deviation from planarity is found for C9 lying $0.029(1) \AA$ from the plane defined by the heterocyclic moiety. In addition, the angle deviation between the benzofuranyl system and a benzene ring is found at $3.26(14)^{\circ}$. This quasiplanar moiety was probably due to an intramolecular hydrogen bond (Table 3) between $\mathrm{C}(5)-\mathrm{H}(5)$ and $\mathrm{O}(10)$. The
Table 3 Hydrogen-bonding geometry $\left(\AA,^{\circ}\right)$

\begin{tabular}{cccc}
\hline $\mathrm{C}-\mathrm{H} \cdots \mathrm{A}$ & $\mathrm{H}-\mathrm{A}$ & $\mathrm{C}-\mathrm{A}$ & $\mathrm{N}-\mathrm{H} \cdots \mathrm{A}$ \\
\hline $\mathrm{O}(8)-\mathrm{H}(8) \cdots \mathrm{O}(18, \mathrm{III})$ & 0.82 & $2.722(3)$ & 156.4 \\
$\mathrm{O}(18)-\mathrm{H}(18) \cdots \mathrm{O}(8, \mathrm{IV})$ & 0.82 & $2.734(3)$ & 167.2 \\
$\mathrm{C}(5)-\mathrm{H}(5) \cdots \mathrm{O}(10)$ & 0.93 & $2.758(3)$ & 156.4 \\
\hline
\end{tabular}

Symmetry codes: III $(-x, 1-y, 1-z) ; \operatorname{IV}(1 / 2+x, 1 / 2-y,-1 / 2+z)$.

$\mathrm{C}(6)-\mathrm{C}(9)-\mathrm{O}(10)$ valence angle at $\mathrm{C}(9)$ was noticed at $115.18(14)^{\circ}$ slightly less than the $120^{\circ}$ usually observed for classical planar $\mathrm{C}\left(\mathrm{sp}^{2}\right)$.

The final atomic coordinates and equivalent isotropic thermal parameters are listed in Table 2. There are no solventaccessible voids in the crystal lattice. Molecules are stabilized in the crystal lattice by hydrogen bonding (Table 3 ).

\section{Acknowledgements}

G. D. Kapche thanks the "Agence Universitaire pour la Francophonie (AUF)" for a six month travel grant to the University Victor Segalen Bordeaux 2.

\section{References}

1. C. C. Berg, J. C. A. Weerdenburg, and M. E. E. Hijman, in "Moracées (incl. Cécropiacées). Flore du Cameroun. Vol. 28”, ed. C. C. Berg, M. E. E. Hijman, and J. C. A. Weerdenburg, 1985, Ministère de l'Enseignement Supérieur et de la Recherche Scientifique, Yaoundé.

2. J. Berhaut, in "Flore illustrée du Sénégal. Dicotylédones. Tome IV”, ed. Gouvernement du Sénégal, Ministère du Développement Rural et de l'Hydraulique, 1979, Direction des Eaux et Forêts, Dakar, 111.

3. L. Qionghong, Z. Guogang, and D. Shuhua, Shenyang Yaoke Daxue Хиеbao, 2003, 20, 386.

4. http://cancer.gov/clinicaltrials/CCUM-2004-0535.

5. Enraf-Nonius CAD-4 Software, Version 5.0, 1989, EnrafNonius: Delft, The Netherlands.

6. G. M. Sheldrick, SHELXS 97, Program Crystal Structure Solution, 1997, University of Göttingen, Germany.

7. G. M. Sheldrick, SHELXL 97, Program for Crystal Structure Refinement, 1997, University of Göttingen, Germany. 